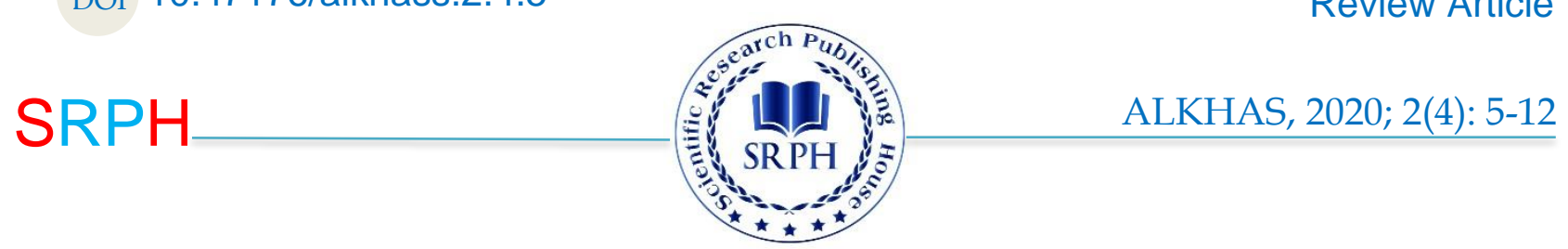

\title{
Investigation of Waste in Livestock and Poultry Industry and Methods to Improve Feed Conversion Ratio in it
}

Seyyed Mohammad Ziaei ${ }^{*}$ and Masoomeh Amini ${ }^{2}$

1Doctor of Veterinary Medicine Intern.

${ }^{2}$ Doctor of Veterinary Medicine Student.

\section{*Corresponding Author: \\ $\rightarrow$ email}

Received: 20 September, 2020

Accepted: 15 November, 2020

Published: 10 November, 2020

\begin{abstract}
Poultry farming has become a lucrative industry to produce a reasonably priced protein food source. But studies show that the most waste in this industry is food waste that appears at an undesirable conversion ratio. In the present study, technical, technological and managerial factors affecting these wastes have been investigated. For this purpose, descriptive and analytical research methods were used. The results showed that among the three factors studied, technical factors include high poultry capacity, lack of official activity license, not using feed and chopped berries; Technological factors include preparing food from one source, especially from informal sources, improper use of the entrance disinfection pond and hygrometer in the halls, using the window in the combined hall ventilation system, incorrect use of the hall disinfection technologies; Finally, managerial factors include a long interval between two hatcheries (leading to fewer hatches per year), an increase in the number of chicks in a rearing period, an early delivery of chickens to the slaughterhouse, and ignorance of managers. The standard of feed waste, excessive feeding and improper distribution of feed in the hall, have affected the high feed conversion ratio. Suggested solutions to reduce food waste are presented in this article.
\end{abstract}

Keywords: Poultry industry, Broiler units, Food waste, Feed conversion ratio

\section{Statement of the problem}

The rate of conversion of food into chicken meat is a major factor in determining the usefulness of raising poultry. The feed conversion ratio (FCR) or the amount of feed required to add one unit of weight is a common indicator used by poultry farmers to assess the relative efficiency of feed [1]. The main effective factors in this field include Physiological factors, management factors, poultry feed and feed items, the effect of feed additives as well as disease control and mortality in poultry farms. Are discussed.

The rate of conversion of food into chicken meat is a major factor in determining the usefulness of raising poultry. The feed conversion ratio (Feed Conversion Ratio) or the amount of feed required per unit weight to add one unit to the live weight of poultry is a common indicator used in the poultry industry to assess the relative performance of feed used by breeders and companies.

\section{Background research}

Nasr et al. (2011) investigated the effect of diet type on the performance of broiler hens of Arian breed. In this experiment, the first factor consisted of two levels of apparent and actual metabolizable energy corrected for nitrogen and the second factor consisted of two levels of total amino acid and digestible feed consumed. The results showed that the percentage of production and egg mass weight and feed conversion ratio in the main effects of energy had a significant difference $(\mathrm{P}<0.05)$. Amiri Andy et al. (2009) investigated the effects of different levels of vitamin $\mathrm{E}$ in broiler diet on egg production and quality. The results of this study showed that experimental diets had no significant effect $(p<0.05)$ on egg production in the whole period but the control group was less than the others. The relationship between nutrition and a fertile's optimal hatching rate is influenced by its stored nutrients and environmental conditions. Age also affects fertility. The idea is that the rooster may take on some of the 
physical properties that gas exchange water has during slaughter. Maternal status of vitamins is often cited as a major factor. But now clear or too much imbalance can leave the fetus alive. Lopez Leeson [2] showed that the dietary chicken protein levels of this significant methionine lysine remained the same as the energy level and remained only $12 \%$ of the single, completely variable raw material. The researchers' complication was the apparent effect of the cause of body weight; Because some were reduced to less by the end of the experiment. Nekofard et al. (2012) examined different meat factors of head 308 strain. The results showed an upward trend during 2003-2009. More precise control and raising the biosafety coefficient of the farm Completion of more severe quarantine entry guidelines led to herd conflict Reduced losses Reduced further research sought Increased health alongside positive management. Predicting consumption usage There are significant changes, while the breeding company to give. The number of birds is directly transmitted growth. The selection should be tailored to individual farms. When the species is assumed to need a bird 21 to 24 weeks of time, it varies, although not clearly, most breeders take the stage of sexual differentiation. The length of the Nimcheh River is about 450 grams. Physical maturity is the most important factor in performance. They can become young. Research on unbalanced amino acid use is a tool of speed. Unfortunately, the size difference is probably a reflection of genetic variance, especially in adults, usually 150-170 meals a day, depending on the ambient temperature of 16, 25 times a day. The theory is relatively small, Leson was able to give a good ability 10 by rich study seen small proportion of data represent to provide a reason for needs such as nutrition. Benefits also include improving sperm volume. Direct result of the optimal vital program. The ability to incubate common infertility. The presence of the reasons for the number of failed matings. Therefore, maintaining their continuous potential is great. Qaisari (2005) Arian Energy, sulfur obtained (zero 49 days) Diet, $(\mathrm{P}<0.05)$ Diet energy had no significant effect on feed intake, feed conversion ratio and carcass components. The results of some experiments also show that increasing the level of protein in the diet, even if it does not increase growth, will improve feed efficiency. In this case, the effect of protein on improving the feed conversion ratio is probably due to the increase in protein content, followed by carcass moisture in parallel with the reduction of fat (12). Golzar Adabi et al. (2007) tested the effect of L-carnitine and vegetable fat powder on the sperm quality of roosters and the fertility and hatching of broilers. The results showed that all-carnitine increased fertility compared to the control group $(\mathrm{P}<0.05)$. Egg characteristics were not affected by diet. The percentage of egg production in the fifth and sixth weeks of the experiment in the groups fed with al-carnitine was higher than the other groups $(\mathrm{P}<0.01)$. Also, the sperm characteristics in the group fed with al-carnitine improved. In general, this experiment showed that the consumption of Lcarnitine in the diet of broiler flocks increases fertility, hatching percentage and sperm quality. In a similar experiment conducted by Newman et al. (2000), sperm concentrations were higher in the third and fourth weeks in all-carnitine-fed cows than in the control group.

\section{Purpose}

Investigation of technical, technological and managerial factors affecting poultry industry waste.

\section{Hypothesis}

It seems that among the three factors studied, technical factors have the most impact on poultry industry waste. In many cases, researchers use an inverse ratio called food efficiency (Feed Efficiency), meaning the weight gain per unit weight per unit of food consumed, to measure the effect of various experimental methods on birds. Numerous factors affect the feed conversion ratio or feed efficiency.

Factors influencing this field and methods of improving the feed conversion ratio are:

Factors related to poultry nutrition.

\section{The amount of energy in the diet}

Animals consume food to meet their energy needs. Therefore, the amount of energy in broiler diets has a direct effect on the amount of food consumed daily by birds [3]. Poultry fed a diet containing $2900 \mathrm{~kg} / \mathrm{kcal}$ consume about $10 \%$ more food than birds fed a diet containing $3300 \mathrm{~kg} / \mathrm{kCal}$, so that dietary energy deficiency and feed intake are a direct and predictable relationship when the diet is fully fed. In terms of protein, amino acids and other essential nutrients are regulated to meet the needs for growth through daily consumption of these substances [4].

It is possible that the amount of energy calculated through the food composition tables is not exactly in accordance with the actual energy of the diet and depending on the type and quality of food consumed is more or less than the actual amount of energy. Usually in food composition tables the energy value of foods of animal origin such as meat, bones and poultry products is estimated to be less than the actual energy produced from these materials.

For example, for a food item containing good quality meat and bones, the energy value is considered to be equivalent to $1980 \mathrm{KG} / \mathrm{KCAL}$, while research at the University of Georgia suggests that the energy value of the above food is close to $2500 \mathrm{KG} / \mathrm{KCAL}$. The 
reason for considering lower energy from food or animal origin is that when determining the metabolizable energy (Metabolisable Energy) of these materials by conventional methods, it is necessary to eat large amounts of food and due to the high amount of calcium and phosphorus content of this Substances are impaired in fat absorption and produce less energy. The use of large amounts of animal products of animal origin in the regulation of the diet of broilers may lead to a better nutritional coefficient, but failure to consider the exact amount of energy of these components can lead to problems.

In the late 1970s, a major problem called Oily Bird Syndrome became common in Georgia poultry due to the relatively cheap price of animal foods compared to soy. The use of the above products up to $15 \%$ of the diet and also considering the low metabolic energy of these substances leads to an increase in the ratio of calories: protein in the diet and this leads to increased fat stores and the problem of fatty bird syndrome. Fat supplementation is one of the foods that probably has the most impact on the energy changes of broiler diets. Fat supplements are added to food exclusively to increase energy levels, but there are huge differences in the quality of these ingredients. This is especially true of supplements called mixed fats, which are sometimes of very poor quality.

\section{The shape and volume of the feed}

Comparing flour grains with each other, the grain that has more volumetric weight causes faster growth of birds [5]. Don pellets also lead to better FCR because less energy is used to feed the bird with pellets. One of the effective factors in feed conversion ratio in broiler breeding is the quality of pelleted feed that is fed to birds. There is a major difference in the growth rate and feed conversion ratio of birds fed pelleted and non-pelleted feed. The reason for this discrepancy is the large amounts of energy expended by birds fed on non-pelleted food [6].

It is estimated that for every $10 \%$ increase in the crushing of pelleted food, the feed conversion ratio increases by one tenth. Although the quality of pelleted food may look good immediately after leaving the mill, this quality should actually be present when the bird eats in the poultry house. The author's observations in poultry farms indicate that efforts should be made to improve the quality of pelleted feed when passing through a feeder [7].

\section{Feeding program}

Research shows that eating according to a schedule improves the feed conversion ratio. According to such a program, broilers receive enough food in four to six meals a day and will not be fed for a short time and less than an hour after the available food is finished. Lack of access to food stimulates the appetite the next time food is delivered to the salon. Although the principles of feeding according to the schedule are very clear, but this requires great care and proper management. If the birds stay hungry for a long time, it will reduce their performance. Eating enough to feed the chickens at a time is essential to this method of proper light regime.

\section{Quality of food storage and waste}

Maintaining the quality of poultry feed in storage and special yachts is very important. Protection against oxidation, mold and contamination are important in maintaining the quality of poultry feed. Keeping the food distribution system clean and in the hall regularly and preventing food wastage by adjusting the level of food is very important in obtaining the appropriate conversion factor [8].

It is quite clear that the amount of food transported to the poultry house that is not consumed directly by the birds is effective in increasing the feed conversion ratio. Undoubtedly, today, due to the improvement of mechanical feeding systems with previous years, food waste is considered as a less valuable factor than other factors affecting the feed conversion ratio. But the question is, how much food waste is really in commercial poultry farming systems? It is very difficult to measure food waste.

Birds that receive relatively pelleted fodder (Pelleted Feeds) are likely to lose some of the food in the litter, which is inedible and covered by poultry house management [9].

\section{Nutritional management of broiler mother herd}

The purpose of nutrition management in broilers is to provide a range of balanced diets that are able to meet the needs of different stages of breeding and production of broilers to achieve maximum production of quality chickens. Maintaining good uniformity and even being as close as possible to weight goals are the main pillars of broiler nutrition. Composer feed, nutrition management as well as general herd management are considered in evaluating the performance of laying hens. Raw materials for broiler feed should be of good quality, predictable and of uniform nutritional value, free of any microbial, toxic and pathogenic contaminants. The hen can be successfully fed with flour, crumble or plate grain, provided that the grain is of good quality and proper nutrition management is applied in each case [10]. In practice, the supply of nutrients to the mother hen is controlled through the combination of diet and the amount of grain consumed. In addition to environmental factors, receiving daily rations in the form of energy, amino acids and other nutrients determine the performance of the herd. 


\section{The main factors affecting the feed conversion ratio of broilers}

Importance of temperature in poultry farms: The most important factor affecting the feed conversion ratio is the temperature of the breeding environment. Birds are warm-blooded, meaning they maintain a relatively constant body temperature despite the ambient temperature.

In cold environments, broilers will eat more but consume large amounts of calories from feed to maintain a normal body temperature. This energy is used to warm the body and is not converted into meat. Optimal temperatures allow broilers to use nutrients beyond the temperature setting for growth. Ambient temperatures have become ideal for increasing feed conversion [11].

At high ambient temperatures, broilers consume less feed and convert it more efficiently. The biological cooling mechanisms that birds use during hot weather are as energetic as the cooling mechanisms used by them during cold weather. In addition, when birds eat food, their body temperature rises due to the metabolic process that takes place during digestion. For this reason, broilers should not be fed in the hottest part of the day (morning to afternoon) on very hot days. Feeding early in the morning and in the evening (when it is cooler) will help to improve the feed conversion ratio and minimize losses [12].

Ventilation and temperature are interdependent. In most cases, increased ventilation causes the room temperature to be lower. Ventilation is sometimes required to maintain the ideal room temperature [13]. Unfortunately, growers often ventilate less in cold weather due to lower fuel costs. it's wrong. Fresh clean air is just as important for growing broilers as fresh feed and water. Ammonia and other toxic gases accumulate in less ventilated halls during the colder months of the year. Studies have shown that feed conversion can be adversely affected by levels of 25 ppm ammonia (this level of ammonia can be detected by the human nose. Experts strongly recommend that broiler breeders be ventilated to remove ammonia in winter. Ventilation will vary depending on indoor insulation, humidity, litter conditions, etc. In the first week, at least 0.10 cubic feet of air per minute is sufficient for each chick, but this should increase as the birds grow. Whenever you feel ammonia, immediately increase the amount of ventilation.

\section{Feed quality of broilers}

The diet that chickens consume mainly affects the feed conversion ratio. You have little control over the energy level, protein or initial quality of the feed (as these are controlled by a nutritionist). However, you must maintain the quality of the food when it is delivered to you and protect it from oxidation, mold and contamination [14]. At the end of each period, wash and disinfect the feed tanks. Never leave food in the tank or feeding system at the end of the period. Check the nutrition system daily to make sure it is working properly. Carefully inspect the areas where the feed may be placed and spoiled. Feed levels should be high in the feed so that the chickens can access it; However, as the chicks grow, reduce the feed level in the feed to prevent birds from spilling out of the feed. Also, as the chicks grow, raise the feeders so that the feeding edge is aligned with the bird's back [15].

Quality of drinking water in poultry: Fresh and clean water is important for feed conversion. Broiler yields on farms with contaminated water are almost always below average. Performance usually improves when you eliminate water pollution. Some experts believe that closed drinking systems improve feed conversion ratio.

One of the reasons for this improvement is water quality. Water in buckets and hangers is exposed to dust, bedding, feed and feces. For this reason, water in these open systems usually contains large amounts of bacteria. High levels of bacteria can cause poor absorption, diarrhea and disease. Water in closed systems is protected against contamination.

Although a lot of effort is required, water can be stored in clean and hygienic open drinking fountains. It is not enough to pour old water on the bed. Drinkers should be washed and disinfected daily. Closed drinking systems do not completely eliminate the daily routine of cleaning drinking fountains. Regardless of the type of drinking water you use, flush the water supply system thoroughly before the chicks arrive so that the chicks have access to fresh water from the beginning.

Water is the most important nutrient for any creature. Your efforts to provide clean water for broilers will lead to better feed conversion.

Elimination: Feed is wasted by sick and disabled birds. Birds that do not have a chance to reach the slaughter weight should be removed as soon as possible. This will improve feed conversion. In terms of disease and health, removal is also reasonable.

Disease and treatment in poultry farms: The general health of the herd affects feed conversion. Carefully monitor the early signs of the disease and treat sick birds appropriately and promptly. Use vaccines and medications carefully because reactions caused by improper use can negatively affect weight gain and feed conversion [16].

When broilers are infected with parasitic worms, the use of anthelmintics is essential. However, these drugs negatively affect feed conversion. Some breeders teach how and when to examine their birds for worms and treat them only when the flocks are in trouble. The use of these drugs is unnecessary when there is no worm and causes a harmful effect on the growth and conversion of food. Temperature, ventilation and 
hygiene, as well as litter conditions, rodent and beetle control; Affects feed conversion.

\section{Other factors affecting the conversion of broiler feed conversion}

Scheduled feeding in poultry: Research shows that timed feeding or meals can improve feed conversion. With such feeding programs, broilers are fed 4 to 6 times a day. When the feed is finished, the chickens do not have access to food for a short time (less than an hour). The short period of not having access to food is apparently to stimulate nutrition at the next meal. Birds are usually calm during this short period, which increases feed conversion.

Although the principle of timed nutrition is correct, it requires careful monitoring and proper management. If the time of not having access to food is prolonged, its benefits are wasted and performance is actually reduced. In this feeding system, more food is needed to feed all or most of the birds at the same time. Do not use scheduled feeding in the last week of growth as it can cause gastrointestinal upset and problems at the time of slaughter.

Effect of light on chickens' feed: Light levels in broiler halls can affect feed conversion. High light (1 to 2 feet of candlelight) stimulates the chickens' activity and helps them find water and food. After 10 to 14 days, light levels can gradually decrease to approximately 0.5 feet of candlelight in the darkest areas of the hall. Low levels of light calm birds and reduce their activity, resulting in better weight gain.

In controlled halls, scheduled feeding using an optical system can enhance feed conversion. A light program with one hour of light and two hours of darkness throughout the day can improve feed conversion.

Birds respond well to good management. Birds raised in a quiet, stress-free environment produce better than birds raised in a stressful environment. Excessive stress causes the birds to expend energy, resulting in growth and feed conversion.

Benefits of an artificial mother:

- Chickens can find their comfort zone.

- The room temperature can be kept below ideal without adversely affecting the performance and health of the chicken -.- If the temperature drops, the stress on the chicken when using the artificial mother is less than the hot air oven method.

- The use of an artificial mother is more suitable for old, outdated, poorly managed halls than a hot oven.

Disadvantages of using an artificial mother:

- Because artificial mothers cover a small space of the bed, they are needed in large numbers for a nursery, this increases the initial costs of maintenance.

Factors affecting feed conversion ratio:
1. Pellet feed with Hygienizer processing and double shaft mix technology

2. Lighting program

3. Seed program

4. Hall temperature

According to scattered studies in poultry farms in Iran, the level of productivity and efficiency in Iran is lower than the standard, because the waste is too reasonable at different stages. In order to clarify the situation of waste and its causes, the waste of poultry industry can be divided into three periods or stages as follows:

\section{Waste in the production stage}

In order to realize the low efficiency and consequently the waste at this stage, an acceptable standard should be in hand to compare with it to evaluate the efficiency of poultry farming in the production stage. In terms of meat production of commercial strains that exist in Iran, their production status has been examined and it is as follows:

Average weight at 8 weeks $1700 \mathrm{~g}$, feed conversion ratio of $2.2 \mathrm{~kg}$, losses of $5.2 \%$. In terms of egg production, studies have shown that under suitable conditions in commercial poultry farms, the productivity and efficiency are as follows. Spawning age 5 months, average 255 eggs, average egg weight $4.60 \mathrm{~g}$ feed-to-egg conversion ratio 7.2 losses in the growing season $5.4 \%$ losses in the laying stage $3.10 \%$ of total losses from The first day until the end of spawning $8.14 \%$. Losses in our country, both in broilers and in laying hens, are more than the standard cultivars, so that according to the available evidence and studies, the average loss of laying hens during breeding is $12 \%$ and in the laying period $18 \%$ and a total of ' 30 . Percentage has been mentioned. Thus, the number of losses in egg flocks in our country is twice the standard.

\section{Food waste}

As mentioned at the beginning, the standard ratio of food to meat is a maximum of 2.2 and the ratio of food to egg is 7.2. While in Iran, preliminary studies have shown that the feed conversion ratio to poultry meat is about 2.5 to 7.2 and the feed to egg ratio is $3 \mathrm{~kg}$. Thus, we have consumed $300-500$ grams per kilo of meat and 300 grams of extra food per kilo of eggs. The presence of disease in poultry flocks often reduces feed efficiency by $3-7 \%$. The causes of waste in Iran can be seen both directly and indirectly. Factors that directly affect can be classified as wastage. This is often Due to incorrect methods of food storage, food distribution, inadequate feeding, the presence of birds and insects, lack of awareness and compassion of workers is so expensive that due to not paying attention to these simple points, often between $5-7 \%$ of food in public poultry farms may be lost. 
Indirect wastage of food (thrower) In addition to the direct wastage of food due to food spoilage, it may also be wasted indirectly, thus reducing the feed conversion ratio and feed efficiency. These causes are briefly as follows:

Low feed efficiency due to disease The presence of disease in poultry flocks often reduces feed efficiency by $3-7 \%$, for example in coccidiosis of parasitic worms after flock treatment. Because the gastrointestinal tract is injured and complications occur in the epithelial cells of the intestine, the intestines can not digest and absorb food after recovery. As a result, a significant amount of food that enters the gastrointestinal tract is excreted without use in the feces. Food efficiency decreases.

Per capita cost related to chicken: If the price of oneday-old chicken is / / 50 Rials and we offer grilled chicken in the weight of one kilogram to the market, in terms of costs related to buying chicken in one kilogram of live weight 50 Rials and if in the weight of 1.5 Let's send 35 Rials per kilogram to the market, and if the same broiler is sent to the market weighing 2 kilograms, the per capita cost of chicken will be 25 Rials per kilogram of meat produced, and considering this issue, a significant figure will be reduced in terms of reducing production costs. On the other hand, it will save on the production of day-old chicks.

Labor-related per capita cost: Chickens are usually more difficult to care for in the first few weeks and require more care and attention, while older chickens require less care and work. On the other hand, because breeding grilled chickens at older ages can keep a smaller number of series per year, so in terms of operations to enter the day-old chickens and also send broilers to the market will need less work to disinfect the nest, therefore The amount of work in the production unit is reduced and reduces the per capita cost of labor.

Fuel costs: Usually, artificial heat is needed in the first five weeks, while fuel costs are eliminated at older ages. Therefore, the more young chickens are sent to the market, the higher the per capita cost and fuel consumption will be when the chickens are sent to the market at older ages and at higher weights. Because there is no need for fuel costs during additional maintenance.

Vaccination and medicine costs Losses:

Given that two Newcastle disease vaccinations are recommended for broilers, both of which are at a young age, the cost of vaccination and medication will be the same for lower weight chickens and higher weight chickens. As a result, the per capita cost of vaccination per unit weight is cheaper for heavier chickens. In terms of mortality, according to studies, most mortality in broilers is in the first few weeks of life, so in general, the percentage of mortality per unit weight in heavy broilers is lower than lighter grilled chickens.

\section{Chicken litter costs}

Because litter is usually used only once for each series of chickens, bedding costs are the same for light and heavy chickens. Thus, the per capita cost of litter to produce one kilogram of meat in heavy chickens is much cheaper than light chickens.

Food costs: The efficiency of converting feed into meat decreases as the chicken ages, but this reduction in efficiency is not so great that it is not economically viable. This varies depending on the breed, the type of food and the way it is fed. Usually, because the quality of the diet is changed at an older age and the percentage of protein is reduced, the diet is cheaper, so to some extent, the low cost of the diet compensates for the reduction in food efficiency. Therefore, the closer the broilers sent to the market are close to the standard weight (1700 g), the less need there is for dayold chicks, fuel, litter, medicine, vaccines, and work, and vice versa, if this issue is not observed, the waste of these materials Will be more [17].

Waste in the exploitation stage: in the case of broilers, this stage includes packing and marketing to the market and, if necessary, storage in the cold store, and in the case of laying hens, it includes the collection of eggs, grading and marketing. The waste that exists at this stage is due to religion:

Egg breaking lesions: According to studies, about 7.3\% of the total egg produced is broken and unusable. Religion breaks down by 3.6 percent on the farm and 3.7 percent by grading, transporting, and shipping. Preliminary studies in Air.

Decreased food efficiency due to not formulating or improperly formulating food: Another point that greatly reduces the efficiency of food is the inappropriate composition of the diet. Increasing and decreasing the proportions set for the poultry diet both reduces feed intake. Existence of lesions due to nonobservance of dietary restrictions in egg halves.

Decreased feed efficiency due to lack of attention to the percentage of production: If a uniform feed is provided to the chicken flock throughout the laying period without regard to the percentage of production, the feed efficiency will decrease, because if the mercury is uniform, for example based on $15 \%$ protein in When the percentage of production is low (less than 65\%), more protein is provided to the chicken, which causes food loss and is not economical. Conversely, when the production is high, the amount of protein is not enough. Therefore, because the needs of the chicken are not fully met, the percentage of production decreases, which in any case reduces the efficiency of food, so depending on the percentage of laying, the necessary changes in He gave a diet and unfortunately such a thing is not common in Iran. Thus, the factors mentioned above cause food efficiency to decrease and waste to increase. Waste from sending low-weight 
chickens to the slaughterhouse: The question of at what age and at what weight grilled chicken is sent to the market is an important factor in terms of waste. Usually the best economic weight for sending chickens to the market is an average weight of 1800 grams. In this weight, maximum productivity is achieved, while in Iran, in practice, the weight of chickens sent to the market is much less than this.

Waste in different stages: In this stage, the waste includes poultry waste, which unfortunately is not used as it should be and maybe in Iran. These lesions are summarized as follows:

Remnants of incubator factories: Incubator factory residues are usually composed of the eggs of hatched eggs, unfertilized eggs, dead sperm eggs, dead embryo eggs and hatched and lost chickens, in addition to some chickens. The killer who sells the laying hens sexually throws the roosters away. A large amount of rooster chicks is added to this waste for a day. In Iran, this waste is thrown away and even a lot of money is spent to destroy it. According to studies, incubator powder contains $27 \%$ protein, $21 \%$ calcium, $0.65 \%$ phosphorus and a large amount of other nutrients that can be easily used to feed laying hens.

Waste residues of poultry slaughterhouses: At the time of slaughter, there are usually $3 \%$ of the head and $5 \%$ of the legs and in general $22 \%$ of the residues, and if we count the feathers as part of these residues, $23 \%$ of the carcass includes the residues. The powder of these residues usually contains $70 \%$ of protein, which is an excellent source of lysine, cysteine and methionine, and its fat content is about $15 \%$ [16]. The percentage of powder compounds in slaughterhouse residues is as follows: Humidity 9.3, protein 71.5 , ether extract 15.2 , hydrocarbons 0.5 , gray 3.5 and metabolic energy per $1720 \mathrm{~kg}$. In terms of amino acid, its components are as follows: arginine 4.55 , cysteine 3.15 , glycine 6.22 , hysteine 1.15 , leucine 5.85 , isoleucine 3.2 , lysine 2.93 , TT4.41. Unfortunately, despite the severe lack of protein raw materials in poultry diets, no use Some of these residues are not due to the lack of equipped slaughterhouses in Iran and are in fact considered waste, while the powder of these materials in terms of nutrition can easily replace much of the imported fish meal.

\section{Conclusion}

Due to the fact that the nutrition management of broilers in different breeding periods in each farm is very important, so knowing the problems of nutrition management in broiler farms is possible through the review of scientific research. Therefore, by knowing the weaknesses and comparing the performance of the herd with the standard of each breed of broiler, it can help to improve the performance in breeding and production periods in broiler flocks. Chickens and roosters of the mother herd, regardless of their breed, should be raised in such a way as to obtain the desired feed efficiency because feed accounts for about $60 \%$ of the cost of raising heifers, but this does not mean that the cheapest feeding program should be used to raise heifers. Because such a program will undoubtedly reduce the production of the mother herd.

Flock feed conversion ratio is economically important for broiler producers. Some of the factors affecting feed conversion ratio are temperature, ventilation, feed quality and water. Broiler breeders who manage their flocks to optimize these factors for better feed conversion will be rewarded for their efforts at the end of the year. For best results, broilers need more food until they are 42 days old. Deciding on the amount of final feed depends on the age of the target weight at the time of slaughter. The final feed is more important in terms of total feed intake and the cost of broiler feed. Therefore, it should be considered for optimal financial return.

As the chicks get older, their activity decreases and they prefer to rest for a long time, and the energy expended on physical activity decreases. During this period, despite consuming more food, due to the slow growth rate, the amount of nutrients required per gram of body weight decreases.

\section{References}

1. Asfari AA. Causes of waste in the country's poultry farms. QJ Livest Aquat Aff. 1373; 5: 22-25.

2. Lopez G, Leeson S. Response of broiler breeder to low-protein diets. 1. Adult breeder performance. Poult Sci. 1995; 74: 685-695.

3. Amin M. The position of management and human resources specialized in the poultry industry. J Poult Indust. 2009; 47-51.

4. Cravener TL, Roush WB, Mashaly MM. Broiler production under varying population densities. Poult Sci. 1992; 71: 427-433.

5. Bakhshizadeh M. Productivity and waste of poultry feed. Marraeh Magazine. 1997; 10: 43-41.

6. Beyer RS. The impact of feed milling and manufacturing procedures on nutrient availability and the importance of quality control. Kansas State University, Department of Animal Science and Industry. 2005; Email: sbeyer@oznet.ksu.edu

7. Neuman SL, Lin T, Hester PY. The effect of dietary carnitine on semen traits of white leghorn roosters. Poult Sci. 2000; 81: 495-500.

8. Moradi M, Maghsoudloo S, Rostami R, Mostafa Lou Y. The effect of different levels of replacement of extruded soybeans instead of soybean meal and different levels of vitamin $\mathrm{E}$ on production index and economic characteristics of broilers. Livest Res. 2012; 1(4): 15-25. 
9. Butcher Gary D, Richard D. Miles. Minimizing microbial contamination in feed mills producing poultry feed. University of Florida IFAS Extension. 2003; http://edis.ifas.ufl.edu/VM054

10. Khodaei H, Maghsoudloo S, Gharehbash AM, Taraz Z. Study of the effect of physical form of feed and probiotic and prebiotic supplements on the performance and characteristics of broiler carcasses. Livest Res. 2015: 6(12) : 20-29.

11. Mountain Therapy, Hassan and Ehud Naji. The position of poultry breeding in the national economy. $J$ Rural Jihad. 1391; 339: 12-13.

12. Zohri MA. Causes of waste in Iranian poultry farms and some suggestions. Iran J Poult. 1978; 3-10.

13. Chapman HD, Johnson ZB, McFarland JL. Environment and Health. Improvements in the performance of commercial broilers in the USA: Analysis for the years 1997 to 2001. Poult Sci. 2003; 82: 50-53.

14. Anonymous. Broiler sample profile. 2005; http://www.fao.org/WAIRDOCS/LEAD/X6170E

/x6170e3g.htm
15. Salahi Moghadam R, Maghsoudloo S, Mostafa Lou Y, Shahir MH, Bayat Kouhsar J. The effect of different protein levels and type of diet on the production and economic performance of broilers weighing approximately one kilogram. Livest Poult Res. 2014; 2(4): 59-70.

16. Deaton JW. The effect of early feed restriction on broiler performance. Poult Sci. 1995; 74: 1280-1286. 24 Fairchild BD. 2005.

17. Cable MC, Waldroup PW. Effects of dietary protein level and length of feeding on performance and abdominal fat content of broiler chickens. Poult Sci. 1991; 70: 1550-1558.

18. Alsobayel AA. Effect of protein rearing diet and age on fertility and hatchability parameters of Saudi Arabian Baladi chickens. J King Saudi Univ. 1999; 4: 4754.

19. Brake J, Anderson KE. Dietary calcium after broiler breeder egg production. Poult Sci. 2005; 74: 106-110.

\section{ALKHAS}

Copyright: (C) 2020 The Author(s); This is an open-access article distributed under the terms of the Creative Commons Attribution License (http://creativecommons.org/licenses/by/4.0), which permits unrestricted use, distribution, and reproduction in any medium, provided the original work is properly cited.

Citation: Ziaei SM, Amini M. Investigation of Waste in Livestock and Poultry Industry and Methods to Improve Feed Conversion Ratio in it. ALKHAS. 2020; 2(4): 5-12.

https://doi.org/10.47176/alkhass.2.4.5 